\title{
Discussion on Teaching Method of the Hydraulic Mechanical Automatic Transmission
}

\author{
LI Mingsheng \\ Institute of Mechatronic Engineering \\ Lingnan Normal University \\ Zhanjiang 524048 China \\ 1097338932@qq.com \\ SUN Yuechao \\ Institute of Mechatronic Engineering \\ Lingnan Normal University \\ Zhanjiang 524048 China
}

\author{
GONG Manfeng \\ Institute of Mechatronic Engineering \\ Lingnan Normal University
Zhanjiang 524048 China \\ Lingnan Normal University
Zhanjiang 524048 China
}

LI Man

Institute of Mechatronic Engineering

Lingnan Normal University

Zhanjiang 524048 China

\begin{abstract}
In the teaching of hydraulic mechanical automatic transmission, the lever method and reduced graph method are used respectively for the teaching of planetary mechanism shift schedule and hydraulic control circuit which are difficult for students to grasp. The lever method is used to develop the tachogram of planetary gears, and the transmission ratio of Simpson and Ravigneaux planetary gears is analyzed. The hydraulic transmission basic circuit analysis method is used in the simplification of hydraulic control circuit of hydraulic mechanical automatic transmission, aiming to solve students' difficulty in the understanding of automatic transmission. It turns out that students master this part very well.
\end{abstract}

Keywords-automatic transmission; planetary gear mechanism; teaching method; discussion

\section{INTRODUCTION}

Hydraulic mechanical automatic transmission is an important part of modern automobile, mainly composed of hydraulic torque converter, planetary gear transmission mechanism, hydraulic control system, electronic control system, cooling oil filter device and shell[1]. The Due to the complexity of the structure and working principle of the automatic transmission, mechanical parts damage, hydraulic system malfunction, or electronic control system failure, will all affect its normal work[2]. At the same time, the automatic transmission is not easy to dismantle, and it has high requirement for assembly environment after dismantling. This has caused great difficulties for the teaching of teachers and the learning, experiment and practice of students. Currently, the teaching materials of automatic transmission in the car service engineering major include "Car Structure", "Vehicle Failure Detection Technology" and "Car Fault Diagnosis And Maintenance". Of which "Car Structure" only introduces automatic transmission components, "Car Fault Detection Technology" introduces the detection method and fault diagnosis method of automatic transmission. But how planetary gear mechanism change gear, and how to understand the

Guangdong province characteristic innovative project (2016KTSCX079), Guangdong province characteristic innovative project (2016GXJK100),

Guangdong province undergraduate college higher education teaching reform project complex automatic transmission hydraulic control oil are not involved in the teaching materials. With years of automatic transmission teaching experience and the combination of practical teaching and research results, the author used the lever method[3] to establish the tachogram of planetary gear mechanism, and analyzed the transmission ratio of Simpson and Ravigneaux[4] planetary gear mechanism at different gears, which are widely used currently; The hydraulic transmission basic circuit[5] analysis method is used in the simplification of hydraulic control circuit of hydraulic mechanical automatic transmission, aiming to solve students' difficulty in the understanding of automatic transmission.

\section{The General Rule of GeAr Shift of Automatic TRANSMISSION PlanETARY GEAR MECHANISM}

In order to meet the multi-gear drive, modern automotive automatic transmission (AT) generally uses the planetary mechanism arrangement of pairs of planetary gear row in parallel with two degrees of freedom, or three and four planetary gear rows in series and parallel with three degrees of freedom. The transmission ratio conversion can be achieved through the separation and engagement of the clutch or brake. Transmission ratio is usually calculated through analytic method and graphic method. The analytic method means to set up the ternary simultaneous equation of planetary gear rows, determine the relationship of rotational speeds in the equation, and input and output components and the common components in each row. First, solve the (braked) single-row equation with the speed of 0 and obtain the equation with the main function of common components. Then, the equation is substituted into the common component of the other equation, thus the duplex equation can be turned into the single row equation with only input and output speed. The input speed is put on the left side of the equal sign, the output speed is put on the right side of the equal sign, so as to solve the equation of the relationship of input shaft speed and output shaft speed, then it is divided by the speed to obtain the speed ratio of input shaft and output 
shaft. The movement characteristic equation of one bank planetary gear mechanism[4] is:

$$
n_{1}+\kappa n_{2}-(1+\kappa) n_{H}=0
$$

The movement characteristic equation of double planetary gear mechanism[4] is:

$$
n_{1}+(\kappa-1) n_{2}-\kappa n_{H}=0
$$

Where: $n_{1}, n_{2}, n_{H}, \kappa$ refer to the sun wheel speed, ring gear speed, planetary carrier speed and gear ratio of gear ring and sun gear respectively.

The algorithm of equation (1) or (2) is relatively simple for single and double row planetary gear transmission analysis, but it is difficult to use the algorithm in analysis of multi-row planetary gear transmission, especially the judging of transmission ratio amount that the mechanism may achieve, its basic components, the relative speed of the planetary gear, and mechanical and dynamic problems. If using the lever method (graphic method) for analysis, the above problem will become very easy for students to understand.

\section{A. Lever Diagram of Single-planetary Gear Transmission}

A planetary gear set is composed of sun gear, planet gear, ring gear and planet carrier. According to its structure characteristics, a planet row is equivalent to a vertical lever and three fulcrums, which respectively represent the sun gear R, planet carrier $\mathrm{H}$ and ring gear $\mathrm{R}$. With regard to the singleplanetary planet row and from formula (1) and conservation of energy theorem, the conclusion that the intermediate fulcrum is planet carrier $\mathrm{H}$ and two-end fulcrums are $\mathrm{S}$ and $\mathrm{R}$ can be obtained; with regard of dual-planetary planet row and from formula (2) and conservation of energy theorem, he conclusion that the intermediate fulcrum is ring gear $\mathrm{R}$ and two-end fulcrums are $\mathrm{H}$ and $\mathrm{S}$ can be obtained. The length between fulcrum $\mathrm{S}$ as well as the length between fulcrum $\mathrm{H}$ and fulcrum $\mathrm{R}$ and fulcrum $\mathrm{H}$ are inversely proportional to the number of sun gear and the number of ring gear. As shown in Fig. 1 a) and $b$ ), they are the lever diagram of single-planetary planet row and dual planetary gear row. $\mathrm{K}$ in this diagram is the ratio of the number of ring gear and sun gear. It is able to analyze the rotational speed of planetary gear by using the level diagram through the following steps: creating a coordinate system and drawing three fulcrums proportionally on y-axis. $\mathrm{X}$-axis represents the size and direction of rotational speed of fulcrum. Here we supposing the rotational speed of the fulcrum is 1 , the direction toward $\mathrm{x}$-axis is positive, otherwise is negative and the speed of the fixed fulcrum is 0 . As shown in Fig. 2 a) and b), Fig. a) is the rotational speed analysis diagram of single-planetary gear. Ring gear R is input. Sun gear S is fixed and planet $\mathrm{H}$ is output. By adopting the Similar Triangles, the rotational speed of the planet carrier $\mathrm{n}_{\mathrm{H}}=\kappa \mathrm{n}_{\mathrm{R}} /(\kappa+1)$ belongs to deceleration and in same direction, which is as same as the computed result of the formula (1); Fig. b) is the rotational speed analysis diagram of dual planetary gear. Planet carrier H is input. Sun gear S is fixed and ring gear R is output. By adopting the Similar Triangles, the rotational speed of the planet carrier $n_{R}=(\kappa-1) n_{H} / \kappa$ belongs to deceleration and in same direction, which is as same as the computed result of the formula (2). But the lever method is easier and more visual.

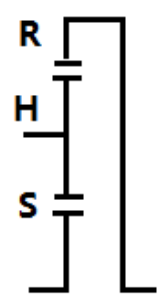

a ) Single-planetary Gear

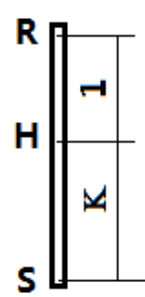

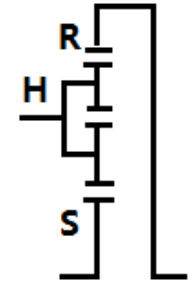

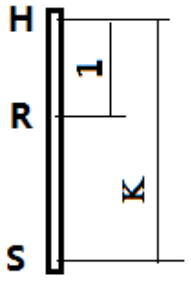

b) Dual Planetary Gear Row
Fig. 1. Lever Diagram of Single-planetary Gear Mechanism
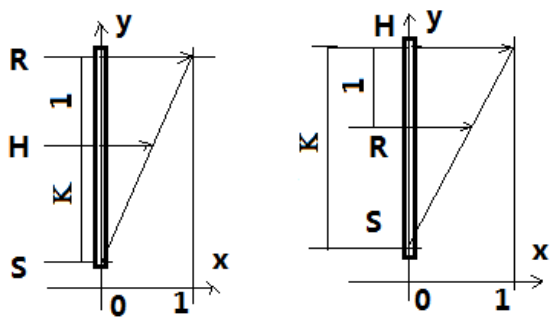

a ) Single-planetary Gear b) Dual Planetary Gear Row

Fig. 2. Rotational Speed Diagram of Single-planetary Gear Mechanism

\section{B. Lever Diagram of Dual-planetary Gear Transmission}

When more than one planet rows are in parallel, each planet row can be regarded as a vertical lever and three fulcrums. The connection of each fulcrum on the lever diagram is able to represent the interconnection of the components between planet rows. While processing, the lever can be overlapped or separated. When they are overlapped, the interconnected parts can be combined into one point. When the lever is separates, the joinpoint can be represented by a horizontal line. In all these two situations, the arm of force of the lever should be adjusted, through which the length of the arm of force among the merge point of connection part should be ensured to be same. The length breadth ratio of each arm of force of lever is same, thus the lever diagram of multiple planet rows can be combined into one general diagram. Mark clutch C, brake B and one-way clutch $\mathrm{F}$, input link $\mathrm{i}$ and output link o on the diagram. Thus the lever diagram of dual-planetary gear transmission appears [6]. Fig. 3 showed is Ravigneanx planetary gear rotational speed diagram. Fig. a) is the structure diagram of Ravigneanx planetary gear; $b$ ) is the speed change diagram of Ravigneanx planetary gear. When connecting K1B1, 1st-Gear appears. When K1B1 is intersecting with R1R2, the length of the intersection segment is the output speed, and its direction is as same as the input speed. In the same way, when connecting K1B2, 2nd-Gear appears. When connecting K1K3, 3rd-Gear appears. When connecting K3B2, 4th-Gear appears. When connecting K2B1, Back gear appears. The transmission ratio of each gear can be got by adopting the method of similar triangles. 

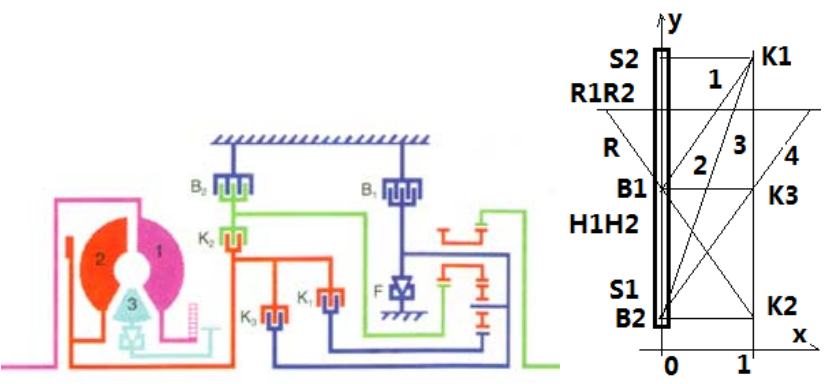

a) structure diagram

b) speed change diagram

Fig. 3. Ravigneanx Planetary Gear Rotational Speed Diagram
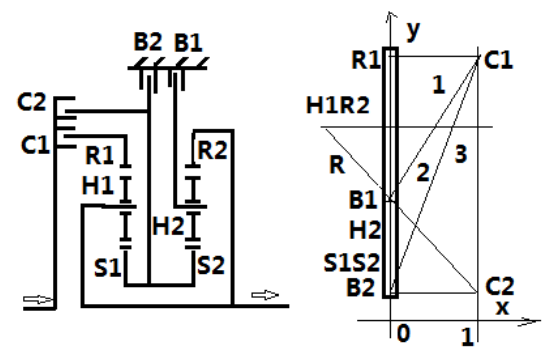

b) schematic diagram

b) speed change diagram

Fig. 4. Simpson Planetary Gear Rotational Speed Diagram

Fig. 4 showed is Simpson planetary gear rotational speed diagram. Fig. a) is the a) schematic diagram of Simpson planetary gear; b) is the speed change diagram of Simpson planetary gear. When connecting C1B1(or F), 1st-Gear appears. When C1B1 is intersecting with R1R2, the length of the intersection segment is the output speed, and its direction is as same as the input speed. In the same way, when connecting C1B2, 2nd-Gear appears. When connecting C2C1, 3rd-Gear appears. When connecting C2B1, R-Gear appears.

The advantage of lever method is that it is easy to distinguish the transmission route of each gear among the complicated compound planetary gear shift mechanism which is composed of three planet rows or four planet rows. For example, the ZF9 automatic transmission newly designed by German ZF Friedrichshafen AG. Its core transmission structure is four groups series-to-parallel planetary gear (the first two planet rows are in parallel connection and the last two planet rows are in parallel connection. While the second and third planet rows in middle are in series connection) and six shifting components, which has realized nine forward gears. And the structural composition can be showed in Fig. 5.

Since ZF9 Automatic Transmission is four-row planetary gear hybrid mechanism and in order to make the lever diagram more clear, the lever speed change diagram can be divided into first two-row and last two-row planet mechanism, as shown in Fig.6 and Fig. 7. The first two-row is Ravigneaux mechanism, in which a planet carrier and a ring gear are shared. Planet carrier is output link, and equivalent lever has four construction points. The mechanism is controlled by two clutches $(\mathrm{B}, \mathrm{F})$ and two brakes (C, D). And it has two degrees freedom, the combination of F_C, F_D can realize two down-shifts. The combination of $\mathrm{F}-\mathrm{B}$ can realize a direct gear. The combination of S_D can realize a back gear. The combination of C_D can realize a mechanism brake. Three forward gears and a reverse gear can be realized, as shown in Fig. 6.

The last two-row is Simpson mechanism with two sun gear connected and the planet carrier in the third row is connecting with the ring gear in the fourth row. The ring gear in the third row connected with the Ravigneaux mechanism is output link, so as the planet carrier in the fourth row. There are four construction points in the equivalent lever, which is controlled by one clutch $\mathrm{E}$ and one brake $\mathrm{A}$. The mechanism has two degrees freedom. The first two-row Ravigneaux mechanism is connected with the ring gear in the third planet row directly. There are four speed points and one braking point. These four speed points are combined with brake $A$ and clutch $E$ respectively, through which eight gears can be realized. With the two gears of E_A_F and E_C_D, another two gear can be realized. The last two-row Simpson mechanism can realize nine forward gears and one reverse gear, as showed in Fig. 7.

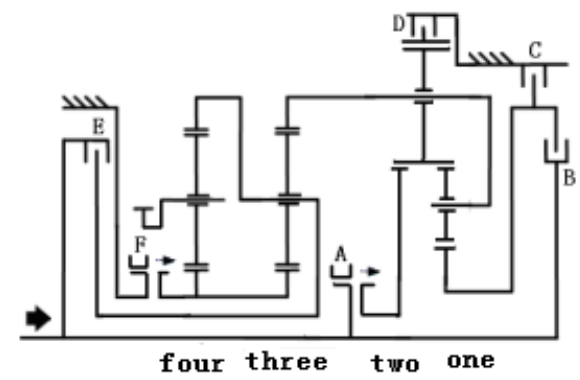

Fig. 5. Structure Diagram of ZF 9 Automatic Transmission

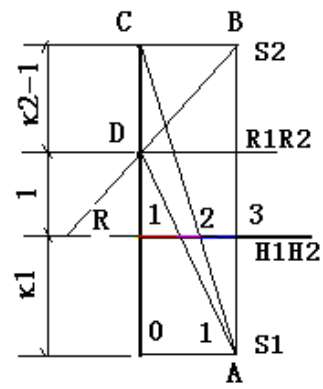

Fig. 6. Ravigneaux Lever Speed Change Diagram

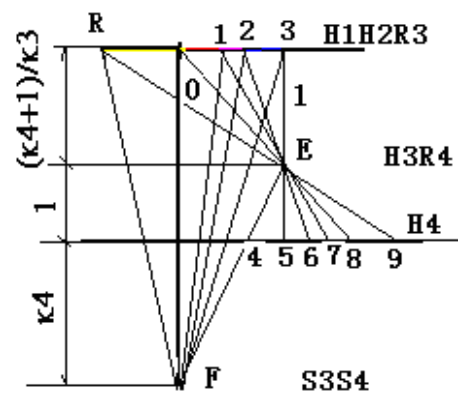

Fig. 7. Simpson Lever Speed Change Diagram 


\section{SiMPLIFICATION OF AUTOMATIC TRANSMISSION HYDRAULIC CONTROL CIRCUIT}

Automatic transmission hydraulic control circuit is used to meet the operational need of automatic transmission. Usually it contains three main circuits of different pressures, which is used to realize the shifting gear of high-pressure oil circuit by controlling the clutch and brake; providing the mediumpressure oil circuit used in the lubrication of torque converter and planetary gears; providing the low-pressure oil circuit which controlled various of hydraulic valve and improving the shifting quality, as the red, yellow and green parts showed in Fig. 5. Taking the simplification of realizing the shift of highpressure oil circuit by controlling clutch and brake as example, it is composed of fluid drive mechanism: hydraulic pump which provides pressure; hydraulic cylinder (clutch and brake) which is able to do the mechanical work; main pressure regulator valve which used to adjusting the systematic pressure (the working pressure of automatic transmission is changing with the element of loading and speed. It is a variate, which can be showed in the figure); control clutch; shift valve which is combined with brake (because clutch and brake are the singleoutput hydraulic cylinder, the organization of selector valve is two-position three-way solenoid valves; compensated flow control valve is used to control the time of shifting (the oneway restrictive valve is mostly used); Energy accumulator which is used to providing the harshness while in shifting (Fig. 8 refers to $\mathrm{K} 3$ coordinate valve), fuel tank and filter. Based on the upper analysis, Fig. 9 is the simplification of the control circuit of the clutch $\mathrm{K} 3$ in Fig. 8. The control circuit of the clutch and brake in same transmission are almost same. The analysis and fault diagnosis analysis of the control circuit on other clutch and brake can be done based on Fig. 9.

\section{CONCLUSION}

(1) While in teaching, the working principle of lever method and the drawing of the analysis diagram of the rotational speed of single-planetary gear should be firstly grasped by students, which is of significance. And it is also the key point to using the lever method.

(2) When drawing the lever diagram of multiple-row planet row gear transmission, the interconnection part can be overlapped into one point. When the lever is separated, the connection point can be represented by a horizontal line; making sure that the length of the arm of force of each merge point of the connection part is same and the ratio of the length of the arm of the force of each lever is same.

(3) When drawing the diagram of hydraulic control circuit, the basic constitution of automatic transmission control circuit should be firstly analyzed. Then based on the composition of hydraulic transmission elementary circuit, simplifying the original element of the automatic transmission control circuit one by one. Thus a control diagram which is easily to be understood is formed.

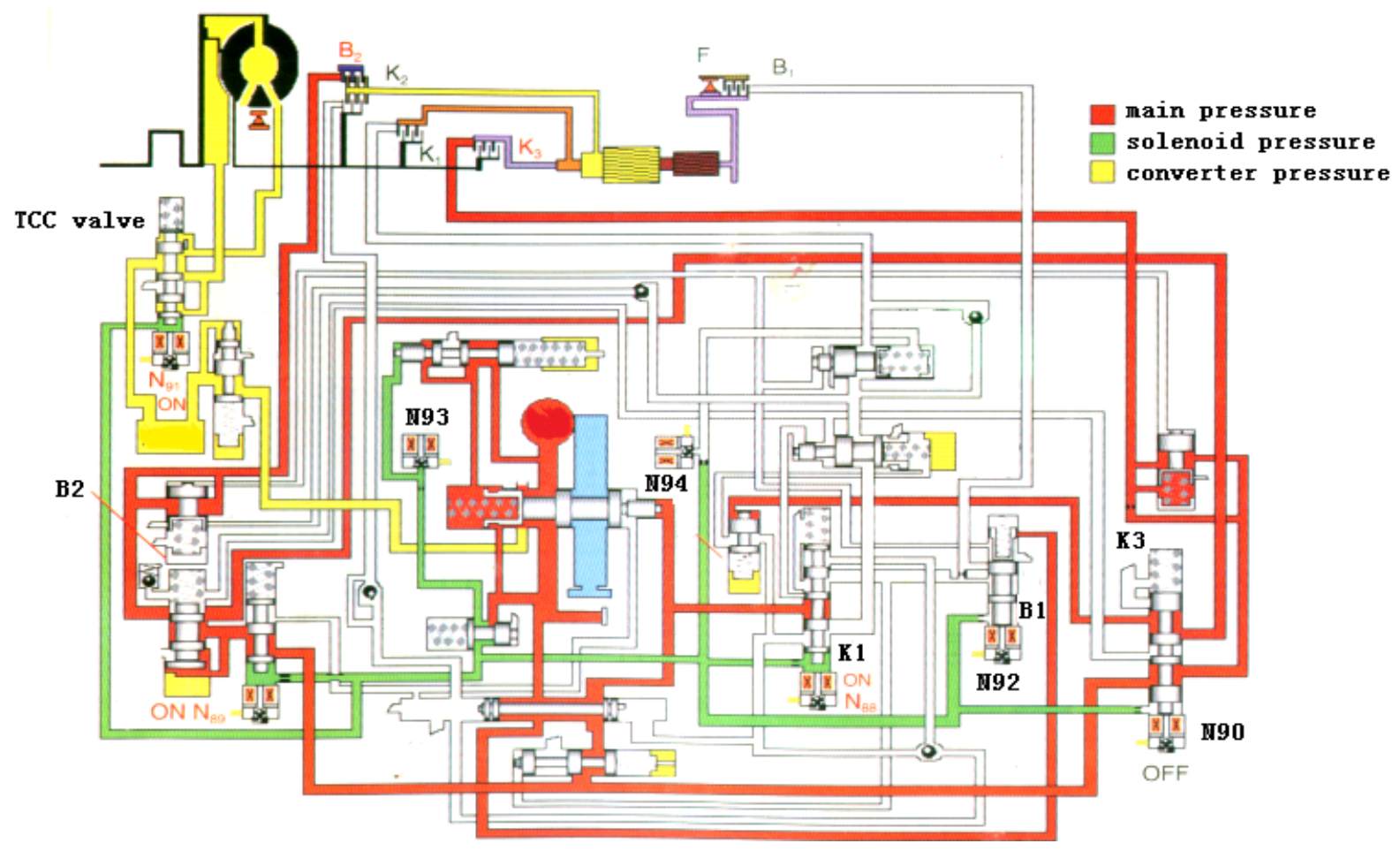

Fig. 8. Volkswagen 01M Automatic Transmission Hydraulic Control Diagram 


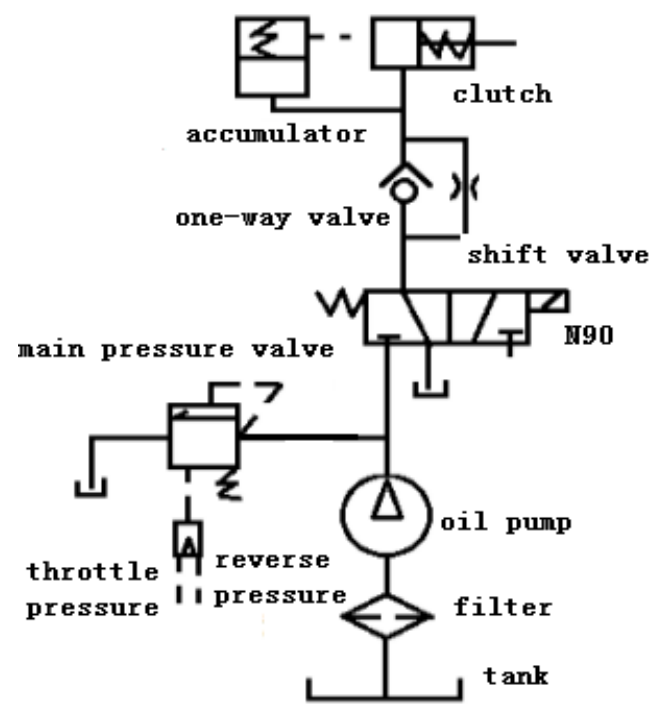

\section{REFERENCES}

[1] Xu Tonghua. Integrated Mode of "Auto Automatic Transmission Repair" Course Teaching [J]. Vehicle Maintenance, 2016 (8): 19-22.(In Chinese)

[2] Chen Qiang. Fault Diagnosis Method of Volkswagen 01M Automatic Transmission [J]. Automobile Parts, 2016 (01): 67-70. (In Chinese)

[3] Benford H L, Leising M B. The Lever Analogy: A New Tool in Transmission Analysis[J]. SAE Technical Paper, 1981:1-9. (

[4] Chen Jiarui, Ma Tianfei. Car structure [M]. Beijing: China Communications Press, 2006. (In Chinese)

[5] Yang Dadong, He Cunxing. Hydraulic and Air Pressure[M] (third edition). Wuhan: Huazhong University of Science and Technology Press, 2008,4. (In Chinese)

[6] Li Xinghua, He Guoqi. Use Equivalent Lever Method to Analyze Planetary Gear Transmission [J]. Mechanical Transmission, 2004 (1): 44-45. (In Chinese)

Fig. 9. 01M Automatic Transmission K3 Clutch System Control Circuit Diagram 